\title{
ON SOLUTION OF TWO-POINT FUZZY BOUNDARY VALUE PROBLEMS
}

\author{
HÜLYA GÜLTEKIN ${ }^{1}$, NIHAT ALTINIŞIK ${ }^{2}$
}

${ }^{1,2}$ Department of Mathematics, Faculty of Arts and Sciences, Ondokuz Mayıs University, 55139,
Kurupelit, Samsun, Turkey

Keywords: Second-order fuzzy differential equations, fuzzy boundary value problems, Hukuhara differentiability.

Abstract: In this paper, we investigate the existence and uniqueness of solutions of two-point fuzzy boundary value problems for second-order fuzzy differential equations. Some sufficient conditions are presented that guarantee the existence and uniqueness of solutions under the approach of Hukuhara differentiability.

\section{Introduction}

Fuzzy set theory is a powerful tool for modeling uncertainly and for processing vague or subjective information in mathematical models [3]. Particulary, the study of fuzzy differential equation forms a suitable setting for mathematical modeling of real world problems in which uncertainties or vagueness pervade. In fact, fuzzy differential equations is a very important topic from theoretical point of view [5], [6], [7], [9] and also it has applications, for example, in population models [4], civil engineering [8], medicine [1], [2].

In this paper, an investigation is made on the solution of two-point fuzzy boundary value problems by using the Hukuhara differentiability. To put it precisely, the two-point fuzzy boundary value problem is given as the form

$$
\begin{gathered}
\text { Ly }:=P_{0}(x) y^{\prime}+P_{1}(x) y^{\prime}+P_{2}(x) y=F(x), x \in(a, b) \\
B_{1}(y):=A y(a)+B^{\prime}(a)=\left[k_{1}\right]^{\alpha} \\
B_{2}(y):=C y(b)+D^{\prime}(b)=\left[k_{2}\right]^{\alpha}
\end{gathered}
$$

where $\left[\mathrm{k}_{1}\right]^{\alpha}=\left[\underline{\mathrm{k}}_{\alpha_{\alpha}}, \overline{\mathrm{k}}_{1_{\alpha}}\right],\left[\mathrm{k}_{2}\right]^{\alpha}=\left[\underline{\mathrm{k}}_{\alpha}, \overline{\mathrm{k}}_{2_{\alpha}}\right]$ are symmetric triangular fuzzy numbers, $\mathrm{A}^{2}+\mathrm{B}^{2} \neq 0$, $C^{2}+D^{2} \neq 0, P_{0}(x), P_{1}(x), P_{2}(x), F(x)$ are continuous functions and $P_{0}(x)$ has no zeros on $(\mathrm{a}, \mathrm{b})$.

\section{Preliminaries}

In this section, we give some definitions and introduce the necessary notation which will be used throughout the paper.

Definition 2.1. A fuzzy number is a function $u: \square \rightarrow[0,1]$ satisfying the following properties:

$\mathrm{u}$ is normal, convex fuzzy set, upper semi-continuous on $\square$ and $\operatorname{cl}\{\mathrm{x} \in \square \mid \mathrm{u}(\mathrm{x})>0\}$ is compact, where cl denotes the closure of a subset.

Let $\square_{F}$ denote the space of fuzzy numbers. 
Definition 2.2. Let $u \in \square_{F}$. The $\alpha$-level set of $u$, denoted $[u]^{2}, 0<\alpha \leq 1$, is $[u]^{\alpha}=\{x \in \square \mid u(x) \geq \alpha\}$. If $\alpha=0$, the support of $u$ is defined $[u]^{0}=\operatorname{cl}\{x \in \square \mid u(x)>0\}$. The notation $[\mathrm{u}]^{\alpha}=\left[\underline{\mathrm{u}}_{\alpha}, \overline{\mathrm{u}}_{\alpha}\right]$, denotes explicitly the $\alpha$-level set of $\mathrm{u}$. We refer to $\underline{\mathrm{u}}$ and $\overline{\mathrm{u}}$ as the lower and upper branches of u, respectively.

The following remark shows when $\left[\underline{\mathrm{u}}_{\alpha}, \overline{\mathrm{u}}_{\alpha}\right]$ is a valid $\alpha$-level set.

Remark 2.3. The sufficient and necessary conditions for $\left[\underline{u}_{\alpha}, \overline{\mathrm{u}}_{\alpha}\right]$ to define the parametric form of a fuzzy number as follows:

(i) $\underline{u}_{\alpha}$ is bounded monotonic increasing (nondecreasing) left-continuous function on $(0,1]$ and right-continuous for $\alpha=0$,

(ii) $\overline{\mathrm{u}}_{\alpha}$ is bounded monotonic decreasing (nonincreasing) left-continuous function on $(0,1]$ and right-continuous for $\alpha=0$,

(iii) $\underline{\mathrm{u}}_{\alpha} \leq \overline{\mathrm{u}}_{\alpha}, 0 \leq \alpha \leq 1$.

Definition 2.4. For $u, v \in \square_{F}$ and $\lambda \in \square$, the sum $u+v$ and the product $\lambda u$ are defined by $[\mathrm{u}+\mathrm{v}]^{\alpha}=[\mathrm{u}]^{\alpha}+[\mathrm{v}]^{\alpha},[\lambda \mathrm{u}]^{\alpha}=\lambda[\mathrm{u}]^{\alpha}, \forall \alpha \in[0,1]$, where $[\mathrm{u}]^{\alpha}+[\mathrm{v}]^{\alpha \alpha}$ means the usual addition of two intervals (subsets) of $\square$ and $\lambda\lceil u]^{\alpha}$ means the usual product between a scalar and a subset of $\square$. The metric structure is given by the Hausdorff distance

$$
\mathrm{D}: \square{ }_{\mathrm{F}} \times \square_{\mathrm{F}} \rightarrow \square+\cup\{0\},
$$

by

$$
\mathrm{D}(\mathrm{u}, \mathrm{v})=\sup _{\alpha \in[0,1]} \max \left\{\left|\underline{\mathrm{u}}_{\alpha}-\underline{\mathrm{v}}_{\alpha}\right|,\left|\overline{\mathrm{u}}_{\alpha}-\overline{\mathrm{v}}_{\alpha}\right|\right\} .
$$

Definition 2.5. Let $u, v \in \square_{\mathrm{F}}$. If there exist $\mathrm{w} \in \square_{\mathrm{F}}$ such that $\mathrm{u}=\mathrm{v}+\mathrm{w}$, then $\mathrm{w}$ is called the Hukuhara difference of $u$ and $v$ and it is denoted by $u \AA$ v.

Definition 2.6. Let $\mathrm{I}=(\mathrm{a}, \mathrm{b})$ and $\mathrm{F}: \mathrm{I} \rightarrow \square_{\mathrm{F}}$ be a fuzzy function. We say $\mathrm{F}$ is Hukuhara differentiable at $t_{0} \in I$, if there exists an element $F^{\prime}\left(t_{0}\right) \in \square_{F}$ such that, for all $h>0$ sufficiently near to 0 , we have $\mathrm{F}\left(\mathrm{t}_{0}+\mathrm{h}\right) \ddot{A} \mathrm{~F}\left(\mathrm{t}_{0}\right), \mathrm{F}\left(\mathrm{t}_{0}\right) \ddot{A} \mathrm{~F}\left(\mathrm{t}_{0}-\mathrm{h}\right)$ and the limits

$$
\lim _{h \rightarrow 0^{+}} \frac{F\left(t_{0}+h\right) \AA ् A F\left(t_{0}\right)}{h}=\lim _{h \rightarrow 0^{+}} \frac{F\left(t_{0}\right) \AA ् A F\left(t_{0}-h\right)}{h}=F^{\prime}\left(t_{0}\right) \text {. }
$$

Here the limits are taken in the metric space $(\square, \mathrm{F})$.

Theorem 2.7. Let $\mathrm{f}: \mathrm{I} \rightarrow \square$ F be a function and denote $[\mathrm{f}(\mathrm{t})]^{\alpha}=\left[\underline{\mathrm{f}}_{\alpha}(\mathrm{t}), \overline{\mathrm{f}}_{\alpha}(\mathrm{t})\right]$, for each $\alpha \in[0,1]$. If $\mathrm{f}$ is Hukuhara differentiable, then $\underline{f}_{\alpha}(t)$ and $\bar{f}_{a}(t)$ are differentiable functions and $\left[\mathrm{f}^{\prime}(\mathrm{t})\right]^{\alpha}=\left[\underline{\mathrm{f}}_{\alpha}^{\prime}(\mathrm{t}), \overline{\mathrm{f}}_{\alpha}^{\prime}(\mathrm{t})\right]$ 


\section{Fuzzy Boundary Value Problem}

In this section we concern with the existence and uniqueness of solution for the problem (1.1)-(1.3). Theorem 3.1. Let $[\phi(\mathrm{x})]^{\alpha}=\left[\underline{\phi}_{\alpha}(\mathrm{x}), \bar{\phi}_{\alpha}(\mathrm{x})\right],[\psi(\mathrm{x})]^{\alpha}=\left[\underline{\psi}_{\alpha}(\mathrm{x}), \bar{\psi}_{\alpha}(\mathrm{x})\right]$. Let $\Phi$ and $\Psi$ be solutions of $\mathrm{Ly}=0$ such that either $\mathrm{B}_{1}(\phi)=\mathrm{B}_{1}(\psi)=0$ or $\mathrm{B}_{2}(\phi)=\mathrm{B}_{2}(\psi)=0$, then $\left\{\underline{\phi}_{\alpha}, \underline{\psi}_{a}\right\}$ and $\left\{\bar{\phi}_{\alpha}, \bar{\psi}_{\alpha}\right\}$ are linearly dependent.

Proof. Recall that $B_{1}(y)=A y(a)+B y(a)$ and $A^{2}+B^{2} \neq 0$. Therefore, if $B_{1}(\phi)=B_{1}(\psi)=0$ then $A$ and $\mathrm{B}$ are nontrivial solutions of the systems

$$
\begin{array}{lll}
\mathrm{A} \underline{\phi}_{\alpha}(\mathrm{a})+\mathrm{B} \underline{\phi}_{\alpha}^{\prime}(\mathrm{a})=0 & \text { and } & \mathrm{A} \bar{\phi}_{\alpha}(\mathrm{a})+\mathrm{B} \bar{\phi}_{\alpha}(\mathrm{a})=0 \\
\mathrm{~A} \underline{\Psi}_{\alpha}(\mathrm{a})+\mathrm{B} \underline{\Psi}_{\alpha}^{\prime}(\mathrm{a})=0 & \mathrm{~A} \bar{\psi}_{\alpha}(\mathrm{a})+\mathrm{B} \bar{\psi}_{\alpha}(\mathrm{a})=0 .
\end{array}
$$

This implies that

$\underline{\phi}_{\alpha}(\mathrm{a}) \underline{\Psi}_{\alpha}^{\prime}(\mathrm{a})-\underline{\phi}_{\alpha}^{\prime}(\mathrm{a}) \underline{\psi}_{\alpha}(\mathrm{a})=0$ and $\bar{\phi}_{\alpha}(\mathrm{a}) \bar{\psi}_{\alpha}(\mathrm{a})-\bar{\phi}_{\alpha}(\mathrm{a}) \bar{\psi}_{\alpha}(\mathrm{a})=0$.

So $\left\{\underline{\phi}_{a}, \underline{\psi}_{a}\right\}$ and $\left\{\bar{\phi}_{\alpha}, \bar{\psi}_{a}\right\}$ are linearly dependent.

Corollary 3.2. If $\left\{\phi_{\alpha}, \underline{\psi}_{\alpha}\right\}$ or $\left\{\bar{\phi}_{\alpha}, \bar{\psi}_{\alpha}\right\}$ is linearly independent, then

$\mathrm{B}_{1}^{2}(\phi)+\mathrm{B}_{1}^{2}(\psi) \neq 0$ and $\mathrm{B}_{2}{ }^{2}(\phi)+\mathrm{B}_{2}{ }^{2}(\psi) \neq 0$.

Theorem 3.3. The following statements are equivalent; that is, they are all true or all false.

(a) There is a fundamental set $\left\{z_{1}, z_{2}\right\}$ of solutions of Ly $=0$ such that

$$
\mathrm{B}_{1}\left(\mathrm{z}_{1}\right) \mathrm{B}_{2}\left(\mathrm{z}_{2}\right)-\mathrm{B}_{1}\left(\mathrm{z}_{2}\right) \mathrm{B}_{2}\left(\mathrm{z}_{1}\right) \neq 0 .
$$

(b) If $\left\{\mathrm{y}_{1}, \mathrm{y}_{2}\right\}$ is a fundamental set of solutions of Ly $=0$ then

$$
\mathrm{B}_{1}\left(\mathrm{y}_{1}\right) \mathrm{B}_{2}\left(\mathrm{y}_{2}\right)-\mathrm{B}_{1}\left(\mathrm{y}_{2}\right) \mathrm{B}_{2}\left(\mathrm{y}_{1}\right) \neq 0 .
$$

(c) For each continuous function $\mathrm{F}$ and pair of symmetric triangular fuzzy numbers $\left(\left[\mathrm{k}_{1}\right]^{\alpha},\left[\mathrm{k}_{2}\right]^{\alpha}\right)$, the fuzzy boundary value problem (1.1)-(1.3) has a unique solution except the $\alpha$-level set.

(d) The homogeneous boundary value problem

$$
\mathrm{Ly}=0, \mathrm{~B}_{1}(\mathrm{y})=0, \mathrm{~B}_{2}(\mathrm{y})=0
$$

has only the trivial solution $\mathrm{y}=0$.

(e) There is a fundamental set $\left\{z_{1}, z_{2}\right\}$ of solutions of $L y=0$ such that $B_{1}\left(z_{1}\right)=0$ and $B_{2}\left(z_{2}\right)=0$. Proof. We show that (a) $\Rightarrow(\mathrm{b}) \Rightarrow(\mathrm{c}) \Rightarrow(\mathrm{d}) \Rightarrow(\mathrm{e}) \Rightarrow(\mathrm{a})$.

(a) $\Rightarrow(b)$ : Since $\left\{z_{1}, z_{2}\right\}$ is a fundamental set of solutions of Ly $=0$, there are constants $a_{1}, a_{2}, b_{1}, b_{2}$ such that

$$
\begin{aligned}
& \mathrm{y}_{1}=\mathrm{a}_{1} \mathrm{z}_{1}+\mathrm{a}_{2} \mathrm{z}_{2}, \\
& \mathrm{y}_{2}=\mathrm{b}_{1} \mathrm{z}_{1}+\mathrm{b}_{2} \mathrm{z}_{2} .
\end{aligned}
$$


Moreover,

$$
\left|\begin{array}{ll}
\mathrm{a}_{1} & \mathrm{a}_{2} \\
\mathrm{~b}_{1} & \mathrm{~b}_{2}
\end{array}\right| \neq 0
$$

Because if this determinant were zero, its rows would be linearly dependent and therefore $\left\{\mathrm{y}_{1}, \mathrm{y}_{2}\right\}$ would be linearly dependent, contrary to our assumption that $\left\{\mathrm{y}_{1}, \mathrm{y}_{2}\right\}$ is a fundamental set of solutions of Ly $=0$. From (3.4),

$\left[\begin{array}{ll}B_{1}\left(y_{1}\right) & B_{2}\left(y_{1}\right) \\ B_{1}\left(y_{2}\right) & B_{2}\left(y_{2}\right)\end{array}\right]=\left[\begin{array}{ll}a_{1} & a_{2} \\ b_{1} & b_{2}\end{array}\right]\left[\begin{array}{ll}B_{1}\left(z_{1}\right) & B_{2}\left(z_{1}\right) \\ B_{1}\left(z_{2}\right) & B_{2}\left(z_{2}\right)\end{array}\right]$.

Since the determinant of a product of matrices is equal to the product of the determinants of the matrices, (3.1) and (3.5) imply (3.2).

(b) $\Rightarrow(c)$ : Since $\left\{\mathrm{y}_{1}, \mathrm{y}_{2}\right\}$ is a fundamental set of solutions of $\mathrm{Ly}=0$, the general solution of $\mathrm{L} y=\mathrm{F}$ is

$$
[\mathrm{y}]^{\alpha}=\left[\underline{\mathrm{y}}_{\alpha_{\mathrm{p}}}+\mathrm{c}_{1}(\alpha) \mathrm{y}_{1}+\mathrm{c}_{2}(\alpha) \mathrm{y}_{2}, \overline{\mathrm{y}}_{\alpha_{\mathrm{p}}}+\mathrm{c}_{3}(\alpha) \mathrm{y}_{1}+\mathrm{c}_{4}(\alpha) \mathrm{y}_{2}\right]
$$

where $\left[y_{p}\right]^{\alpha}=\left[\underline{y}_{\alpha_{p}}, \bar{y}_{\alpha_{p}}\right]$ is a particular solution of Ly $=F$. To satisfy the boundary conditions, we must choose

$$
\begin{aligned}
& \underline{\mathrm{k}_{1_{\alpha}}}=\mathrm{B}_{1}\left(\underline{\mathrm{y}}_{\alpha_{\mathrm{p}}}\right)+\mathrm{c}_{1}(\alpha) \mathrm{B}_{1}\left(\mathrm{y}_{1}\right)+\mathrm{c}_{2}(\alpha) \mathrm{B}_{1}\left(\mathrm{y}_{2}\right), \\
& \underline{\mathrm{k}_{2}}=\mathrm{B}_{2}\left(\underline{\mathrm{y}}_{\alpha_{\mathrm{p}}}\right)+\mathrm{c}_{1}(\alpha) \mathrm{B}_{2}\left(\mathrm{y}_{1}\right)+\mathrm{c}_{2}(\alpha) \mathrm{B}_{2}\left(\mathrm{y}_{2}\right)
\end{aligned}
$$

and

$\overline{\mathrm{k}_{1 \alpha}}=\mathrm{B}_{1}\left(\overline{\mathrm{y}}_{\mathrm{a}_{\mathrm{p}}}\right)+\mathrm{c}_{3}(\alpha) \mathrm{B}_{1}\left(\mathrm{y}_{1}\right)+\mathrm{c}_{4}(\alpha) \mathrm{B}_{1}\left(\mathrm{y}_{2}\right)$,

$\overline{\mathrm{k}}_{2 \alpha}=\mathrm{B}_{2}\left(\overline{\mathrm{y}}_{\mathrm{c}_{\mathrm{p}}}\right)+\mathrm{c}_{3}(\alpha) \mathrm{B}_{2}\left(\mathrm{y}_{1}\right)+\mathrm{c}_{4}(\alpha) \mathrm{B}_{2}\left(\mathrm{y}_{2}\right)$,

which are equivalent to

$\mathrm{c}_{1}(\alpha) \mathrm{B}_{1}\left(\mathrm{y}_{1}\right)+\mathrm{c}_{2}(\alpha) \mathrm{B}_{1}\left(\mathrm{y}_{2}\right)=\mathrm{k}_{1} \alpha-\mathrm{B}_{1}\left(\underline{\mathrm{y}}_{\alpha_{\mathrm{p}}}\right)$,

$\mathrm{c}_{1}(\alpha) \mathrm{B}_{2}\left(\mathrm{y}_{1}\right)+\mathrm{c}_{2}(\alpha) \mathrm{B}_{2}\left(\mathrm{y}_{2}\right)=\underline{\mathrm{k}_{2}} \alpha-\mathrm{B}_{2}\left(\underline{\mathrm{y}}_{\alpha_{\mathrm{p}}}\right)$

and

$c_{3}(\alpha) B_{1}\left(y_{1}\right)+c_{4}(\alpha) B_{1}\left(y_{2}\right)=\bar{k}_{1_{\alpha}}-B_{1}\left(\bar{y}_{\alpha_{p}}\right)$,

$\mathrm{c}_{3}(\alpha) \mathrm{B}_{2}\left(\mathrm{y}_{1}\right)+\mathrm{c}_{4}(\alpha) \mathrm{B}_{2}\left(\mathrm{y}_{2}\right)=\overline{\mathrm{k}}_{2 \alpha}-\mathrm{B}_{2}\left(\overline{\mathrm{y}}_{\mathrm{a}_{\mathrm{p}}}\right)$.

(c) $\Rightarrow$ (d): Obviously, $y=0$ is a solution of (3.3). From (c) with $F=0$ and $\left[k_{1}\right]^{\alpha}=\left[k_{2}\right]^{\alpha}=0$, it is the only solution.

(d) $\Rightarrow(e)$ : Let $\left\{y_{1}, y_{2}\right\}$ be a fundamental system for Ly $=0$ and let

$\mathrm{z}_{1}=\mathrm{B}_{1}\left(\mathrm{y}_{2}\right) \mathrm{y}_{1}-\mathrm{B}_{1}\left(\mathrm{y}_{1}\right) \mathrm{y}_{2}$ and $\mathrm{z}_{2}=\mathrm{B}_{2}\left(\mathrm{y}_{2}\right) \mathrm{y}_{1}-\mathrm{B}_{2}\left(\mathrm{y}_{1}\right) \mathrm{y}_{2}$. 
Then $\mathrm{B}_{1}\left(\mathrm{z}_{1}\right)=0$ and $\mathrm{B}_{2}\left(\mathrm{z}_{2}\right)$. To see that $\mathrm{z}_{1}$ and $\mathrm{z}_{2}$ are linearly independent, note that

$$
\begin{gathered}
\mathrm{a}_{1} \mathrm{z}_{1}+\mathrm{a}_{2} \mathrm{z}_{2}=\mathrm{a}_{1}\left[\mathrm{~B}_{1}\left(\mathrm{y}_{2}\right) \mathrm{y}_{1}-\mathrm{B}_{1}\left(\mathrm{y}_{1}\right) \mathrm{y}_{2}\right]+\mathrm{a}_{2}\left[\mathrm{~B}_{2}\left(\mathrm{y}_{2}\right) \mathrm{y}_{1}-\mathrm{B}_{2}\left(\mathrm{y}_{1}\right) \mathrm{y}_{2}\right] \\
=\left[\mathrm{B}_{1}\left(\mathrm{y}_{2}\right) \mathrm{a}_{1}+\mathrm{B}_{2}\left(\mathrm{y}_{2}\right) \mathrm{a}_{2}\right] \mathrm{y}_{1}-\left[\mathrm{B}_{1}\left(\mathrm{y}_{1}\right) \mathrm{a}_{1}+\mathrm{B}_{2}\left(\mathrm{y}_{1}\right) \mathrm{a}_{2}\right] \mathrm{y}_{2} .
\end{gathered}
$$

Therefore, since $y_{1}$ and $y_{2}$ are linearly independent, $a_{1} z_{1}+a_{2} z_{2}=0$ if and only if

$$
\left[\begin{array}{ll}
B_{1}\left(y_{1}\right) & B_{2}\left(y_{1}\right) \\
B_{1}\left(y_{2}\right) & B_{2}\left(y_{2}\right)
\end{array}\right]\left[\begin{array}{l}
a_{1} \\
a_{2}
\end{array}\right]=\left[\begin{array}{l}
0 \\
0
\end{array}\right] \text {. }
$$

If this system has a nontrivial solution then so does the system

$$
\left[\begin{array}{ll}
\mathrm{B}_{1}\left(\mathrm{y}_{1}\right) & \mathrm{B}_{1}\left(\mathrm{y}_{2}\right) \\
\mathrm{B}_{2}\left(\mathrm{y}_{1}\right) & \mathrm{B}_{2}\left(\mathrm{y}_{2}\right)
\end{array}\right]\left[\begin{array}{l}
\mathrm{c}_{1} \\
\mathrm{c}_{2}
\end{array}\right]=\left[\begin{array}{l}
0 \\
0
\end{array}\right] \text {. }
$$

This implies that $y=c_{1} z_{1}+c_{2} z_{2}$ is a nontrivial solution of (3.3), which contradicts (d).

(e) $\Rightarrow$ (a): Theorem 3.1 implies that if $\mathrm{B}_{1}\left(\mathrm{z}_{1}\right)=0$ and $\mathrm{B}_{2}\left(\mathrm{z}_{2}\right)=0$ then $\mathrm{B}_{1}\left(\mathrm{z}_{2}\right) \neq 0$ and $\mathrm{B}_{2}\left(\mathrm{z}_{1}\right) \neq 0$. This implies (3.1), which completes the proof.

Theorem 3.4. Suppose the homogeneous boundary value problem (3.3) has only the trivial solution. Let $\mathrm{y}_{1}$ and $\mathrm{y}_{2}$ be a fundamental set of solutions of $\mathrm{Ly}=0$ such that $\mathrm{B}_{1}\left(\mathrm{y}_{1}\right)=0$ and $\mathrm{B}_{2}\left(\mathrm{y}_{2}\right)=0$, and let $W=y_{1} y_{2}-y_{1} y_{2}$. Then the unique solution except the $\alpha$-level set of

$$
\mathrm{Ly}=\mathrm{F}, \mathrm{B}_{1}(\mathrm{y})=[0]^{\alpha}, \mathrm{B}_{2}(\mathrm{y})=[0]^{\alpha}
$$

is

$$
[\mathrm{y}(\mathrm{x})]^{\alpha}=\left[\underline{\mathrm{y}}_{\alpha}(\mathrm{x}), \overline{\mathrm{y}}_{\alpha}(\mathrm{x})\right]
$$

where

$$
\begin{aligned}
& \underline{y}_{\alpha}(x)=y_{1}(x)\left(\int_{x}^{b} \frac{F(t) y_{2}(t)}{P_{0}(t) W(t)} d t+\frac{(-1+\alpha)}{B_{2}\left(y_{1}\right)}\right)+y_{2}(x)\left(\int_{a}^{x} \frac{F(t) y_{1}(t)}{P_{0}(t) W(t)} d t+\frac{(-1+\alpha)}{B_{1}\left(y_{2}\right)}\right), \\
& \bar{y}_{\alpha}(x)=y_{1}(x)\left(\int_{x}^{b} \frac{F(t) y_{2}(t)}{P_{0}(t) W(t)} d t+\frac{(1-\alpha)}{B_{2}\left(y_{1}\right)}\right)+y_{2}(x)\left(\int_{a}^{x} \frac{F(t) y_{1}(t)}{P_{0}(t) W(t)} d t+\frac{(1-\alpha)}{B_{1}\left(y_{2}\right)}\right)
\end{aligned}
$$

and $[0]^{\alpha}=[-1+\alpha, 1-\alpha]$.

Proof. Suppose the solution of Ly=F is

$[\mathrm{y}]^{\alpha}=\left[\mathrm{u}_{1}(\mathrm{x}, \alpha) \mathrm{y}_{1}+\mathrm{u}_{2}(\mathrm{x}, \alpha) \mathrm{y}_{2}, \mathrm{u}_{3}(\mathrm{x}, \alpha) \mathrm{y}_{1}+\mathrm{u}_{4}(\mathrm{x}, \alpha) \mathrm{y}_{2}\right]$. 
Using the method of variation of parameters
$\mathrm{u}_{1}^{\prime}(\mathrm{x}, \alpha) \mathrm{y}_{1}+\mathrm{u}_{2}^{\prime}(\mathrm{x}, \alpha) \mathrm{y}_{2}=0$
$\mathrm{u}_{3}(\mathrm{x}, \alpha) \mathrm{y}_{1}+\mathrm{u}_{4}(\mathrm{x}, \alpha) \mathrm{y}_{2}=0$
$u_{1}^{\prime}(x, \alpha) y_{1}^{\prime}+u_{2}^{\prime}(x, \alpha) y_{2}^{\prime}=\frac{F}{P_{0}}$
and
$\mathrm{u}_{3}(\mathrm{x}, \alpha) \mathrm{y}_{1}+\mathrm{u}_{4}^{\prime}(\mathrm{x}, \alpha) \mathrm{y}_{2}^{\prime}=\frac{\mathrm{F}}{\mathrm{P}_{0}}$

are obtained. Solving for $\mathrm{u}_{1}^{\prime}(\mathrm{x}, \alpha), \mathrm{u}_{2}^{\prime}(\mathrm{x}, \alpha), \mathrm{u}_{3}^{\prime}(\mathrm{x}, \alpha)$ and $\mathrm{u}_{4}^{\prime}(\mathrm{x}, \alpha)$ yields

$u_{1}^{\prime}(x, \alpha)=-\frac{F_{2}}{P_{0} W}, u_{2}^{\prime}(x, \alpha)=\frac{F_{1}}{P_{0} W}, u_{3}^{\prime}(x, \alpha)=-\frac{F_{2}}{P_{0} W}, u_{4}^{\prime}(x, \alpha)=\frac{F_{1}}{P_{0} W}$.

Integrating these yields

$\mathrm{u}_{1}(\mathrm{x}, \alpha)=\int_{\mathrm{x}}^{\mathrm{b}} \frac{\mathrm{F}(\mathrm{t}) \mathrm{y}_{2}(\mathrm{t})}{\mathrm{P}_{0}(\mathrm{t}) \mathrm{W}(\mathrm{t})} \mathrm{dt}+\mathrm{u}_{1}(\mathrm{~b}, \alpha), \quad \mathrm{u}_{2}(\mathrm{x}, \alpha)=\int_{\mathrm{a}}^{\mathrm{x}} \frac{\mathrm{F}(\mathrm{t}) \mathrm{y}_{1}(\mathrm{t})}{\mathrm{P}_{0}(\mathrm{t}) \mathrm{W}(\mathrm{t})} \mathrm{dt}+\mathrm{u}_{2}(\mathrm{a}, \alpha)$,
$\mathrm{u}_{3}(\mathrm{x}, \alpha)=\int_{\mathrm{x}}^{\mathrm{b}} \frac{\mathrm{F}(\mathrm{t}) \mathrm{y}_{2}(\mathrm{t})}{\mathrm{P}_{0}(\mathrm{t}) \mathrm{W}(\mathrm{t})} \mathrm{dt}+\mathrm{u}_{3}(\mathrm{~b}, \alpha), \quad \mathrm{u}_{4}(\mathrm{x}, \alpha)=\int_{\mathrm{a}}^{\mathrm{x}} \frac{\mathrm{F}(\mathrm{t}) \mathrm{y}_{1}(\mathrm{t})}{\mathrm{P}_{0}(\mathrm{t}) \mathrm{W}(\mathrm{t})} \mathrm{dt}+\mathrm{u}_{4}(\mathrm{a}, \alpha)$.

Using $B_{1}(y)=A y(a)+B^{\prime}(a)=[-1+\alpha, 1-\alpha] \quad$ and $\quad B_{2}(y)=C y(b)+D y^{\prime}(b)=[-1+\alpha, 1-\alpha]$, we solved $\mathrm{u}_{1}(\mathrm{~b}, \alpha), \mathrm{u}_{2}(\mathrm{a}, \alpha), \mathrm{u}_{3}(\mathrm{~b}, \alpha), \mathrm{u}_{4}(\mathrm{a}, \alpha)$ as

$\mathrm{u}_{1}(\mathrm{~b}, \alpha)=\frac{(-1+\alpha)}{\mathrm{B}_{2}\left(\mathrm{y}_{1}\right)}, \mathrm{u}_{2}(\mathrm{a}, \alpha)=\frac{(-1+\alpha)}{\mathrm{B}_{1}\left(\mathrm{y}_{2}\right)}, \mathrm{u}_{3}(\mathrm{~b}, \alpha)=\frac{(1-\alpha)}{\mathrm{B}_{2}\left(\mathrm{y}_{1}\right)}, \mathrm{u}_{4}(\mathrm{a}, \alpha)=\frac{(1-\alpha)}{\mathrm{B}_{1}\left(\mathrm{y}_{2}\right)}$.

This completes the proof.

Theorem 3.5. Suppose the homogeneous boundary value problem (3.3) has a nontrivial solution $y_{1}$ and let $y_{2}$ be any solution of $L y=0$ that isn't a constant multiple of $y_{1}$. Let $W=y_{1} y_{2}^{\prime}-y_{1} y_{2}$. If $\alpha=1$, then the fuzzy boundary value problem (3.6) has infinitely many solutions, all of the form $\mathrm{y}=\mathrm{y}_{\mathrm{p}}+\mathrm{c}_{1} \mathrm{y}_{1}$, where

$\mathrm{y}_{\mathrm{p}}=\mathrm{y}_{1}(\mathrm{x}) \int_{\mathrm{x}}^{\mathrm{b}} \frac{\mathrm{F}(\mathrm{t}) \mathrm{y}_{2}(\mathrm{t})}{\mathrm{P}_{0}(\mathrm{t}) \mathrm{W}(\mathrm{t})} \mathrm{dt}+\mathrm{y}_{2}(\mathrm{x}) \int_{\mathrm{a}}^{\mathrm{x}} \frac{\mathrm{F}(\mathrm{t}) \mathrm{y}_{1}(\mathrm{t})}{\mathrm{P}_{0}(\mathrm{t}) \mathrm{W}(\mathrm{t})} \mathrm{dt}$

and $c_{1}$ is a arbitrary constant. Also $[0]^{\alpha}=[-1+\alpha, 1-\alpha]$. If $\alpha \neq 1$, then (3.6) has no solution.

Proof. From the proof of Theorem 3.4, $\left[y_{p}(x)\right]^{\alpha}=\left[\underline{y}_{\alpha_{p}}(x), \bar{y}_{\alpha_{p}}(x)\right]$ is a particular solution of Ly $=F$, where

$$
\begin{aligned}
& \underline{\mathrm{y}}_{a_{\mathrm{p}}}(\mathrm{x})=\mathrm{y}_{1}(\mathrm{x})\left(\int_{\mathrm{x}}^{\mathrm{b}} \frac{\mathrm{F}(\mathrm{t}) \mathrm{y}_{2}(\mathrm{t})}{\mathrm{P}_{0}(\mathrm{t}) \mathrm{W}(\mathrm{t})} \mathrm{dt}+\frac{(-1+\alpha)}{\mathrm{B}_{2}\left(\mathrm{y}_{1}\right)}\right)+\mathrm{y}_{2}(\mathrm{x})\left(\int_{\mathrm{a}}^{\mathrm{x}} \frac{\mathrm{F}(\mathrm{t}) \mathrm{y}_{1}(\mathrm{t})}{\mathrm{P}_{0}(\mathrm{t}) \mathrm{W}(\mathrm{t})} \mathrm{dt}+\frac{(-1+\alpha)}{\mathrm{B}_{1}\left(\mathrm{y}_{2}\right)}\right), \\
& \bar{y}_{a_{\mathrm{p}}}(\mathrm{x})=\mathrm{y}_{1}(\mathrm{x})\left(\int_{\mathrm{x}}^{\mathrm{b}} \frac{\mathrm{F}(\mathrm{t}) \mathrm{y}_{2}(\mathrm{t})}{\mathrm{P}_{0}(\mathrm{t}) \mathrm{W}(\mathrm{t})} \mathrm{dt}+\frac{(1-\alpha)}{\mathrm{B}_{2}\left(\mathrm{y}_{1}\right)}\right)+\mathrm{y}_{2}(\mathrm{x})\left(\int_{\mathrm{a}}^{\mathrm{x}} \frac{\mathrm{F}(\mathrm{t}) \mathrm{y}_{1}(\mathrm{t})}{\mathrm{P}_{0}(\mathrm{t}) \mathrm{W}(\mathrm{t})} \mathrm{dt}+\frac{(1-\alpha)}{\mathrm{B}_{1}\left(\mathrm{y}_{2}\right)}\right) .
\end{aligned}
$$

Therefore the general solution of (3.6) is of the form 
$[\mathrm{y}]^{\alpha}=\left[\underline{\mathrm{y}}_{\alpha_{\mathrm{p}}}+\mathrm{c}_{1}(\alpha) \mathrm{y}_{1}+\mathrm{c}_{2}(\alpha) \mathrm{y}_{2}, \overline{\mathrm{y}}_{\alpha_{\mathrm{p}}}+\mathrm{c}_{3}(\alpha) \mathrm{y}_{1}+\mathrm{c}_{4}(\alpha) \mathrm{y}_{2}\right]$

Then from $B_{1}(y)=A y(a)+B^{\prime}(a)=[-1+\alpha, 1-\alpha]$, we obtained $c_{2}(\alpha)=c_{4}(\alpha)=0$. From $\mathrm{B}_{2}(\mathrm{y})=\mathrm{Cy}(\mathrm{b})+\mathrm{Dy}^{\prime}(\mathrm{b})=[-1+\alpha, 1-\alpha]$, we obtained $\alpha=1$. Therefore $\mathrm{y}=\mathrm{y}_{\mathrm{p}}+\mathrm{c}_{1} \mathrm{y}_{1}$. This completes the proof.

Example 3.6. Consider the fuzzy boundary value problem

$$
x^{2} y^{\prime}-2 x y^{\prime}+2 y=2 x^{3}, y(1)=[3+\alpha, 5-\alpha], y^{\prime}(2)=[2+\alpha, 4-\alpha] \text {. }
$$

Here $B_{1}(y)=y(1)$ and $B_{2}(y)=y^{\prime}(2)$. Let $\left\{y_{1}, y_{2}\right\}=\left\{x^{2}, x\right\}$, which is a fundamental set of solutions of homogeneous differential equation in (3.10). Then

$\mathrm{B}_{1}\left(\mathrm{y}_{1}\right) \mathrm{B}_{2}\left(\mathrm{y}_{2}\right)-\mathrm{B}_{1}\left(\mathrm{y}_{2}\right) \mathrm{B}_{2}\left(\mathrm{y}_{1}\right)=-3$.

So Theorem 3.3 implies that (3.10) has a unique solution except the $\alpha$-level set. The solution of differential equation in (3.10) is

$[\mathrm{y}(\mathrm{x})]^{\alpha}=\left[\mathrm{x}^{3}+\mathrm{c}_{1}(\alpha) \mathrm{x}^{2}+\mathrm{c}_{2}(\alpha) \mathrm{x}, \mathrm{x}^{3}+\mathrm{c}_{3}(\alpha) \mathrm{x}^{2}+\mathrm{c}_{4}(\alpha) \mathrm{x}\right]$.

Using boundary conditions, coefficients $c_{1}(\alpha), c_{2}(\alpha), c_{3}(\alpha), c_{4}(\alpha)$ are obtained as $c_{1}(\alpha)=c_{3}(\alpha)=-4, c_{2}(\alpha)=6+\alpha, c_{4}(\alpha)=8-\alpha$.

Example 3.7. Consider the fuzzy boundary value problem

$$
y+y=1, y(0)=[-1+\alpha, 1-\alpha], y\left(\frac{\pi}{2}\right)=[-1+\alpha, 1-\alpha] \text {. }
$$

Here $B_{1}(y)=y(0)$ and $B_{2}(y)=y\left(\frac{\pi}{2}\right)$. Let $\left\{y_{1}, y_{2}\right\}=\{\sin x, \cos x\}$, which is a fundamental set of solutions of homogeneous differential equation in (3.11). Then

$\mathrm{B}_{1}\left(\mathrm{y}_{1}\right) \mathrm{B}_{2}\left(\mathrm{y}_{2}\right)-\mathrm{B}_{1}\left(\mathrm{y}_{2}\right) \mathrm{B}_{2}\left(\mathrm{y}_{1}\right)=-1$.

So Theorem 3.3 implies that (3.11) has a unique solution except the $\alpha$-level set. Also, the Wronskian of $\left\{\mathrm{y}_{1}, \mathrm{y}_{2}\right\}$ is

$$
\mathrm{W}(\mathrm{x})=\sin \mathrm{x}(-\sin \mathrm{x})-\cos \mathrm{x} \cos \mathrm{x}=-1 \text {. }
$$

Then (3.7), (3.8) and (3.9) yield the solution $[y(x)]^{\alpha}=[(-2+\alpha)(\cos x+\sin x)+1,(-\alpha)(\cos x+\sin x)+1]$.

Example 3.8. If we take

$$
y(0)=[-1+\alpha, 1-\alpha], y(\pi)=[-1+\alpha, 1-\alpha]
$$

as the boundary conditions for the fuzzy boundary value problem in (3.11), we have $\mathrm{B}_{1}\left(\mathrm{y}_{1}\right) \mathrm{B}_{2}\left(\mathrm{y}_{2}\right)-\mathrm{B}_{1}\left(\mathrm{y}_{2}\right) \mathrm{B}_{2}\left(\mathrm{y}_{1}\right)=0$.

The solution of differential equation in (3.11) is $[\mathrm{y}(\mathrm{x})]^{\alpha}=\left[\underline{\mathrm{y}}_{\alpha}(\mathrm{x}), \overline{\mathrm{y}}_{\alpha}(\mathrm{x})\right]$, 
Where

$\underline{y}_{\alpha}(x)=c_{1}(\alpha) \cos x+c_{2}(\alpha) \sin x+1, \quad \bar{y}_{\alpha}(x)=c_{3}(\alpha) \cos x+c_{4}(\alpha) \sin x+1$.

From boundary conditions, we have

$\underline{y}_{\alpha}(0)=-1+\alpha \Rightarrow c_{1}(\alpha)=-2+\alpha, \quad \bar{y}_{\alpha}(0)=1-\alpha \Rightarrow c_{3}(\alpha)=-\alpha$,

$\underline{\mathrm{y}}_{\alpha}(\pi)=-1+\alpha \Rightarrow \mathrm{c}_{1}(\alpha)=2-\alpha, \quad \overline{\mathrm{y}}_{\alpha}(\pi)=1-\alpha \Rightarrow \mathrm{c}_{3}(\alpha)=\alpha$.

Therefore, differential equatian in (3.11) with boundary conditions (3.12) has no solution.

Example 3.9. Consider the fuzzy boundary value problem

$$
y^{*}+y=\sin 2 x, y(0)=[-1+\alpha, 1-\alpha], y(\pi)=[-1+\alpha, 1-\alpha] \text {. }
$$

The solution of differential equation in (3.13) is

$[y(x)]^{\alpha}=\left[c_{1}(\alpha) \cos x+c_{2}(\alpha) \sin x-\frac{\sin 2 x}{3}, c_{3}(\alpha) \cos x+c_{4}(\alpha) \sin x-\frac{\sin 2 x}{3}\right]$.

From boundary conditions, we get

$$
\begin{array}{ll}
\underline{\mathrm{y}}_{\alpha}(0)=-1+\alpha \Rightarrow \mathrm{c}_{1}(\alpha)=-1+\alpha, & \overline{\mathrm{y}}_{\alpha}(0)=1-\alpha \Rightarrow \mathrm{c}_{3}(\alpha)=1-\alpha, \\
\underline{\mathrm{y}}_{\alpha}(\pi)=-1+\alpha \Rightarrow \mathrm{c}_{1}(\alpha)=1-\alpha, & \overline{\mathrm{y}}_{\alpha}(\pi)=1-\alpha \Rightarrow \mathrm{c}_{3}(\alpha)=-1+\alpha .
\end{array}
$$

Therefore, if $\alpha=1,(3.13)$ has infinitely many solutions, differing by constant multiples of sinx. If $\alpha \neq 1,(3.13)$ has no solution.

Example 3.10. Consider the fuzzy boundary value problem

$$
y^{\prime \prime}+y=F(x), y(0)+y^{\prime}(0)=[-1+\alpha, 1-\alpha], y(\pi)+y^{\prime}(\pi)=[-1+\alpha, 1-\alpha] \text {. }
$$

Here $B_{1}(y)=y(0)+y^{\prime}(0)$ and $B_{2}(y)=y(\pi)+y^{\prime}(\pi)$. Let $\left\{z_{1}, z_{2}\right\}=\{\cos x, \sin x\}$, which is a fundamental set of solutions of hompgeneous differential equation in (3.14). Then $\mathrm{B}_{1}\left(\mathrm{z}_{1}\right) \mathrm{B}_{2}\left(\mathrm{z}_{2}\right)-\mathrm{B}_{1}\left(\mathrm{z}_{2}\right) \mathrm{B}_{2}\left(\mathrm{z}_{1}\right)=2$.

So Theorem 3.3 implies that (3.14) has a unique solution except the $\alpha$-level set. Let

$\mathrm{y}_{1}=\mathrm{B}_{1}\left(\mathrm{z}_{2}\right) \mathrm{z}_{1}-\mathrm{B}_{1}\left(\mathrm{z}_{1}\right) \mathrm{z}_{2}=\cos \mathrm{x}-\sin \mathrm{x}$ and $\mathrm{y}_{2}=\mathrm{B}_{2}\left(\mathrm{z}_{2}\right) \mathrm{z}_{1}-\mathrm{B}_{2}\left(\mathrm{z}_{1}\right) \mathrm{z}_{2}=\cos \mathrm{x}+\sin \mathrm{x}$.

Then $\mathrm{B}_{1}\left(\mathrm{y}_{1}\right)=0, \mathrm{~B}_{2}\left(\mathrm{y}_{2}\right)=0$ and the Wronskian of $\left\{\mathrm{y}_{1}, \mathrm{y}_{2}\right\}$ is

$$
W(x)=(\cos x-\sin x)(-\sin x+\cos x)-(\cos x+\sin x)(-\sin x-\cos x)=2 \text {. }
$$

So (3.7), (3.8) and (3.9) yield the solution

$$
[\mathrm{y}(\mathrm{x})]^{\alpha}=\left[\underline{\mathrm{y}}_{\alpha}(\mathrm{x}), \overline{\mathrm{y}}_{\alpha}(\mathrm{x})\right] \text {, }
$$

Where

$$
\begin{aligned}
\underline{y}_{\alpha}(\mathrm{x})= & \frac{\cos \mathrm{x}-\sin \mathrm{x}}{2}\left(\int_{\mathrm{x}}^{\pi} \mathrm{F}(\mathrm{t})(\cos \mathrm{t}+\sin \mathrm{t}) \mathrm{dt}+(1-\alpha)\right) \\
& +\frac{\cos \mathrm{x}+\sin \mathrm{x}}{2}\left(\int_{0}^{\mathrm{x}} \mathrm{F}(\mathrm{t})(\cos \mathrm{t}-\sin \mathrm{t}) \mathrm{dt}+(\alpha-1)\right),
\end{aligned}
$$




$$
\begin{aligned}
\bar{y}_{\alpha}(x)= & \frac{\cos x-\sin x}{2}\left(\int_{x}^{\pi} F(t)(\cos t+\sin t) d t+(\alpha-1)\right) \\
& +\frac{\cos x+\sin x}{2}\left(\int_{0}^{x} F(t)(\cos t-\sin t) d t+(1-\alpha)\right) .
\end{aligned}
$$

\section{References}

[1] M. F. Abbod, D. G. Von Keyserlingk, D. A. Linkens and Mahfouf, Survey of utilisation of fuzzy technology in medicine and healthcare, Fuzzy Sets and Systems, 120 (2001), 331-349.

[2] S. Barro and R. Marn, Fuzzy logic in medicine, Heidelberg: Physica-Verlag, 2012.

[3] Y. Chalco-Cano, H. Roman-Flores, On new solutions of fuzzy differential equations, Chaos, Solitons \& Fractals 38 (2008), 112-119.

[4] M. Guo and R. Li, Impulsive functional differential inclusions and fuzzy population models, Fuzzy Sets and Systems, 138 (2003), 601-615.

[5] O. Kaleva, Fuzzy differential equations, Fuzzy Sets and Systems, 24 (1987), 301-317.

[6] O. Kaleva, A note on fuzzy differential equations, Nonlinear Analysis, 64 (2006), 895-900.

[7] J. J. Nieto and R. Rodriguez-Lopez, Bounded solutions for fuzzy differential and integral equations, Chaos, Solitons and Fractals, 27 (2006), 1376-1386.

[8] M. Oberguggenberger and S. Pittschmann, Differential equations with fuzzy parameters, Math. Mod. Syst., 5 (1999), 181-202.

[9] D. Vorobiev and S.Seikkala, Toward the theory of fuzzy differential equations, Fuzzy Sets and Systems, 125 (2002), 231-237 